\title{
Ant Colony Optimisation approaches for the transportation assignment problem
}

\author{
L. D’Acierno ${ }^{1}$, M. Gallo ${ }^{2}$ \& B. Montella ${ }^{1}$ \\ ${ }^{I}$ Department of Transportation Engineering, \\ 'Federico II' University of Naples, Italy \\ ${ }^{2}$ Department of Engineering, University of Sannio (Benevento), Italy
}

\begin{abstract}
In this paper, we propose an ACO-based algorithm that can be used to simulate mass-transit networks; this algorithm imitates the behaviour of public transport users. In particular, we show that the proposed algorithm, which is an extension of that proposed by D'Acierno et al. (A stochastic traffic assignment algorithm based on Ant Colony Optimisation, Lecture Notes in Computer Science 4150, pp. 25-36, 2006), allows mass-transit systems to be simulated in less time but with the same accuracy compared with traditional assignment algorithms. Finally, we state theoretically the perfect equivalence in terms of hyperpath choice behaviour between artificial ants (simulated with the proposed algorithm) and mass-transit users (simulated with traditional assignment algorithms).

Keywords: Ant Colony Optimisation, traffic assignment models, hyper-path approach, mass-transit system simulation.
\end{abstract}

\section{Introduction}

In analyses of real dimension networks, simulation models need to provide rapid solutions so that a large number of alternative projects may be explored or consequences of a strategy in terms of future (minutes or hours) network conditions may be simulated beforehand. Hence, in this paper we verify the possibility of developing a meta-heuristic algorithm that allows user flows on the mass-transit system to be calculated more quickly than by using traditional algorithms. In particular, we steered our research into ant-based algorithms. Such algorithms, based on the food source search of ant colonies, have in many cases 
shown their efficiency in terms of calculation time (an extended overview of antbased algorithms can be found in Dorigo and Stützle [2]).

An exhaustive review of applications to transportation systems can be found in Teodorovic [3]. However, the main applications were proposed by Poorzahedy and Abulghasemi [4] in the case of network design, by de Oliveira and Bazzan [5] for traffic control, and D'Acierno et al. [1] and Mussone et al. [6] for traffic assignment.

Almost all ACO traffic assignment algorithms proposed in the literature are based on preventive choice user behaviour which is typical of models for simulating road systems. Instead, in the case of transit systems in an urban context, it is necessary to simulate preventive-adaptive choice behaviours because users are not able to set their itineraries preventively. Indeed, since the physical path depends on the arrival of vehicles at boarding stops, users choose the set of attractive lines beforehand (preventive stage) and the line used according to arrival events (adaptive stage).

Therefore, in this paper we propose an ACO algorithm that allows us to simulate preventive-adaptive user behaviour in the case of transit systems. In particular, we show that the proposed algorithm has an MSA framework and state the perfect equivalence in terms of (hyper-)path choice behaviours between artificial ants (with the proposed approach) and mass transit users (simulated with traditional algorithms). Finally, we show numerically that the proposed algorithm can be adopted to solve equilibrium assignment problems in less time but with the same accuracy compared with traditional algorithms.

\section{The ACO approach to the traffic assignment problem}

In the case of real ants the search for food sources is based on the following simple rules: each ant provides a pheromone trail along its path; each ant follows a path if there is a pheromone trail; if there is no pheromone trail, ants choose their paths randomly; there is evaporation of pheromone trails, which causes short paths to have a more intense trail of pheromone; if there is a diversion point (i.e. where different paths start), ants follow the path with the most intense trail of pheromone. Hence, if a path is blocked by an obstacle, ants initially choose their path randomly; then, due to evaporation, they choose to follow the path with the most intense trail of pheromone, which is the shortest path.

Similarly, artificial ants can be described by these simple rules:

- the probability of choosing a path, indicated as transition probability, which depends on the intensity of the pheromone trail and, sometimes, a visibility term which expresses a sort of distance that could affect probability choices, that is:

$$
p^{t}(l \mid i)=\frac{\left(\tau_{l}^{t}\right)^{\alpha} \cdot\left(\eta_{l}^{t}\right)^{\beta}}{\sum_{l^{\prime} \in F S(i)}\left(\tau_{l^{\prime}}^{t}\right)^{\alpha} \cdot\left(\eta_{l^{\prime}}^{t}\right)^{\beta}} .
$$


where: $p^{t}(l / i)$ is the probability of choosing link $l$, with $l=(i, j)$, at diversion node $i ; \tau_{l}^{t}\left[\tau_{l^{\prime}}{ }^{t}\right]$ is the intensity of the pheromone trail on link $l\left[l^{\prime}\right]$ at iteration $t ; \eta_{l}^{t}\left[\eta_{l^{\prime}}{ }^{t}\right]$ is the visibility term on link $l\left[l^{\prime}\right]$ at iteration $t ; F S(i)$ is the set of links belonging to the forward star of node $i ; \alpha$ and $\beta$ are model parameters;

- the pheromone increase is the rule that expresses the quantity produced by each ant at any iteration, that is:

$$
\Delta \tau_{l}^{t}=\lambda_{l}\left(\boldsymbol{p}^{t}, \boldsymbol{X}\right) .
$$

where: $\Delta \tau_{l}^{t}$ is the pheromone trail produced by each ant on link $l$ at iteration $t ; \boldsymbol{p}^{t}$ is the transition probability matrix at iteration $t$ whose generic element is $p^{t}(l / i) ; \boldsymbol{X}$ is the matrix of model parameters; $\lambda_{l}$ is the function that expresses the increase in the pheromone trail on link $l$ depending on $\boldsymbol{p}^{t}$ and $X$

- the pheromone trail update is the rule that expresses how the pheromone trail evaporates and how it is increased by the contribution of each ant, that is:

$$
\begin{gathered}
\tau_{l}^{t}=(1-\rho) \cdot \tau_{l}^{(t-1)}+\rho \cdot \Delta \tau_{l}^{t}, \\
{\left[\tau_{l}^{t}=(1-\rho) \cdot \tau_{l}^{(t-1)}+\Delta \tau_{l}^{t}\right]}
\end{gathered}
$$

where $\rho$ is the evaporation term.

Generally, a traffic assignment problem can be formulated as a fixed point problem which can be obtained by combining the supply model with the demand model. The supply model describes the performances of transportation systems depending on user flows, that is:

$$
\boldsymbol{C}=\boldsymbol{A}^{\mathrm{T}} \boldsymbol{c}(\boldsymbol{f})+\boldsymbol{C}^{N A} .
$$

where: $\boldsymbol{C}$ is the vector of path generalised costs whose generic element $C_{k}$ is the path cost related to path $k ; \boldsymbol{A}$ is the link-path incidence matrix whose generic element $a_{l, k}$ is equal to 1 if link $l$ belongs to path $k, 0$ otherwise; $c$ is the vector of link costs whose generic element $c_{l}$ is the link cost related to link $l ; f$ is the vector of link flows whose generic element $\boldsymbol{f}_{l}$ is the link flow related to link $l$; $\boldsymbol{C}^{\mathrm{NA}}$ is the vector of non-additive path costs whose generic element $C_{k}^{N A}$ is the non-additive path cost of path $k$ (such as: road tolls at motorway entrance/exit points or transit fares).

Likewise, the demand model imitates user choices influenced by transportation system performances, that is:

$$
f=A P(-C) d .
$$

where $\boldsymbol{P}$ is the matrix of path choice probabilities whose generic element $p_{k, o d}$ expresses the probability of users travelling between origin-destination pair od choosing path $k ; \boldsymbol{d}$ is the demand vector whose generic element $d_{o d}$ expresses the 
average number of users travelling between origin-destination pair od in a time unit.

Therefore, the traffic assignment model can be obtained by combining eqn (5) with (6), that is:

$$
\boldsymbol{f}=\boldsymbol{A P}\left(-\boldsymbol{A}^{\mathrm{T}} \boldsymbol{c}(\boldsymbol{f})+\boldsymbol{C}^{N A}\right) \boldsymbol{d} .
$$

The existence and the uniqueness of the (equilibrium) solution of problem (7) may be stated, as shown by Cantarella [7] and Cascetta [8], if:

1. choice probability functions, $\boldsymbol{P}(-\boldsymbol{C})$, are continuous;

2. link cost functions, $\boldsymbol{c}(\boldsymbol{f})$, are continuous;

3. each Origin-Destination pair is connected (i.e. $I_{o d} \neq \Phi \forall \forall$ od);

4. path choice models are expressed by strictly decreasing functions with respect to path generalised costs, that is:

$$
\left[\boldsymbol{P}\left(-\boldsymbol{C}^{\prime}\right)-\boldsymbol{P}\left(-\boldsymbol{C}^{\prime \prime}\right)\right]^{\mathrm{T}}\left(\boldsymbol{C}^{\prime}-\boldsymbol{C}^{\prime \prime}\right)<0 \quad \forall \boldsymbol{C}^{\prime} \neq \boldsymbol{C}^{\prime \prime} .
$$

5. cost functions are expressed by monotone non-decreasing functions with respect to link flows, that is:

$$
\left[c\left(f^{\prime}\right)-c\left(f^{\prime \prime}\right)\right]^{\mathrm{T}}\left(\boldsymbol{f}^{\prime}-\boldsymbol{f}^{\prime \prime}\right) \geq 0 \quad \forall \boldsymbol{f}^{\prime} \neq \boldsymbol{f}^{\prime \prime} .
$$

Cantarella [7] proposed to solve problem (7) by means of a solution algorithm known as the Method of Successive Averages (MSA). In particular, two algorithms, a Flow Averaging (MSA-FA) and a Cost Averaging (MSA-CA) algorithm, were proposed based respectively on the following sequences:

$$
\begin{gathered}
\boldsymbol{f}^{t+1}=\boldsymbol{f}^{t}+(1 / t) \cdot\left(\boldsymbol{f}\left(\boldsymbol{c}\left(\boldsymbol{f}^{t}\right)\right)-\boldsymbol{f}^{t}\right) \text { with } \boldsymbol{f}^{t} \in \boldsymbol{S}_{\boldsymbol{f}} . \\
\boldsymbol{c}^{t+1}=\boldsymbol{c}^{t}+(1 / t) \cdot\left(\boldsymbol{c}\left(\boldsymbol{f}\left(\boldsymbol{c}^{t}\right)\right)-\boldsymbol{c}^{t}\right) \text { with } \boldsymbol{c}^{1}=\boldsymbol{c}\left(\boldsymbol{f}^{1}\right) ; \boldsymbol{f}^{t} \in \boldsymbol{S}_{\boldsymbol{f}} .
\end{gathered}
$$

where $\boldsymbol{f}($.$) is the network loading function described by eqn (6).$

MSA algorithms stop when a stopping criterion is satisfied. In particular, in the case of MSA-FA and MSA-CA the stopping criterion is always:

$$
\max _{l}\left|\frac{f_{l}\left(\boldsymbol{c}\left(\boldsymbol{f}^{t}\right)\right)-f_{l}^{t}}{f_{l}^{t}}\right|<\varepsilon_{M S A} .
$$

where: $f_{l}^{t}$ is the link flow associated to link $l$ at iteration $t ; f_{l}($.$) is the link flow$ function which provides the flow of link $l$ by means of eqn (6); $\varepsilon_{M S A}$ is the threshold used in the stopping criterion of MSA algorithms.

By means of an extension of Blum's theorem (Blum [9]), Cantarella [7] stated the convergence of MSA algorithms described by eqns (10) and (11).

Analysing eqns (10) and (11), we may note that an assignment algorithm can be formulated as a sequence of network loadings. In particular, Dial's algorithm (Dial [10]) is a stochastic network loading algorithm based on the following rules: 
- Let $Z_{d, i}$ be the minimum cost between node $i$ and destination node $d$;

- a link $l=(i, j)$ is considered for a feasible path, indicated as a Dial-efficient path, only if:

$$
Z_{d, i}>Z_{d, j}
$$

- it is possible to associate to each node and each link a numerical quantity, indicated respectively as node weight and link weight, calculated as:

$$
\begin{gathered}
W_{d, d}=1, \\
w_{d,(i, j)}=\left\{\begin{array}{cl}
\exp \left(-c_{(i, j)} / \theta\right) \cdot W_{d, j} & \text { if } Z_{d, i}>Z_{d, j} \\
0 & \text { if } Z_{d, i} \leq Z_{d, j}
\end{array}\right. \\
W_{d, i}=\sum_{(i, h) \in F S(i)} w_{d,(i, h)} .
\end{gathered}
$$

where: $W_{d, i}$ is the node weight associated to node $i$ in the case of destination node $d ; w_{d,(i, j)}$ is the link weight associated to link $(i, j)$ in the case of destination node $d ; c_{(i, j)}$ is the link cost associated to link $(i, j) ; F S(i)$ is the set of links belonging to the forward star of node $i$;

- the probability of choosing link $l=(i, j)$ at diversion node $i$ is equal to:

$$
p(l \mid i)=w_{d,(i, j)} / W_{d, i} .
$$

- the link flow $f_{l}$ of generic link $l$ can be calculated as:

$$
f_{l=(i, j)}=\sum_{d} e_{d,(i, j)}=\sum_{d} E_{d, i} \cdot w_{d,(i, j)} / W_{d, i}
$$

with:

$$
\begin{gathered}
E_{d, i}=\sum_{(h, j) \in B S(j)} e_{d,(h, j)}, \\
E_{d, o}=d_{o d} .
\end{gathered}
$$

where: $B S(j)$ is the set of links belonging to the backward star of node $j ; d_{o d}$ is the average number of users travelling between origin-destination pair od in a time unit.

D'Acierno et al. [1] proposed an ACO algorithm for solving the traffic assignment problem based on the following assumption:

- the initial intensity of the pheromone trail on each link $l$, associated to ant colony $o d$, indicated as $\tau_{o d, l}^{0}$, is a function of path costs, that is:

$$
\tau_{o d, l}^{0}=\sum_{k: l \in k} T_{o d, k}^{0}
$$


with:

$$
T_{o d, k}^{0}=\left\{\begin{array}{cl}
\exp \left(-C_{k}^{0} / \theta\right) & \text { if } k \in I_{o d} \\
0 & \text { if } k \notin I_{o d}
\end{array} .\right.
$$

where $T^{0}{ }_{\text {od, }, k}$ is the initial intensity of the pheromone trail on path $k, C_{k}^{0}$ is the initial cost of path $k, \theta$ is the parameter of path choice model, $I_{o d}$ is the set of all available (or considered) paths that join origin node $o$ with destination node $d$;

- the increase in the pheromone trail, indicated as $\Delta \tau^{t}{ }_{o d, l}$, can be expressed by a function of path costs, that is:

$$
\Delta \tau_{o d, l}^{t}=\sum_{k: l \in k} \Delta T_{o d, k}^{t},
$$

with:

$$
\Delta T_{o d, k}^{t}=\left\{\begin{array}{cl}
\exp \left(-C_{k}{ }^{t} / \theta\right) & \text { if } k \in I_{o d} \\
0 & \text { if } k \notin I_{o d}
\end{array} .\right.
$$

where $\Delta T^{t}{ }_{o d, k}$ is the increase in the pheromone trail at iteration $t$ and $C^{t}{ }_{k}$ is the path $k$ cost at iteration $t$;

- updating of the pheromone trail can be expressed as:

$$
\tau_{o d, l}^{t+1}=(1-\rho) \cdot \tau_{o d, l}^{t}+\rho \cdot \Delta \tau_{o d, l}^{t+1} .
$$

where evaporation coefficient $\rho$ is variable and equal to $1 / t$ (according to the approach proposed by Li and Gong [11].

In particular, D'Acierno et al. [1], who showed that application of ACO theory in the case of the traffic assignment problem can be considered as an 'algorithmic trick' in order to speed up the calculation of the equilibrium solution, stated the theoretical equivalence of the proposed ACO algorithm to the application of an MSA algorithm where the successive averages were applied to the weight of Dial's algorithm (Dial [10]), that is:

$$
\begin{gathered}
w_{d,(i, j)}^{t+1}=w_{d,(i, j)}^{t}+(1 / t) \cdot\left(\Delta w_{d,(i, j)}^{t+1}-w_{d,(i, j)}^{t}\right), \\
W_{d, i}^{t+1}=W_{d, i}^{t}+(1 / t) \cdot\left(\Delta W_{d, i}^{t+1}-W_{d, i}^{t}\right) .
\end{gathered}
$$

by fixing $\tau_{o d, l}^{t+1}=w_{o d, l}^{t+1}$ and $\Delta \tau_{o d, l}^{t+1}=\Delta w_{o d, l}^{t+1}$.

In this case, the MSA stopping criterion was:

$$
\max _{l}\left|\frac{f_{l}\left(\boldsymbol{w}\left(\boldsymbol{c}\left(\boldsymbol{f}^{t}\right)\right)\right)-f_{l}^{t}}{f_{l}^{t}}\right|=\max _{l}\left|\frac{f_{l}\left(\boldsymbol{\tau}\left(\boldsymbol{c}\left(\boldsymbol{f}^{t}\right)\right)\right)-f_{l}^{t}}{f_{l}^{t}}\right|<\varepsilon_{M S A}
$$


where: $\boldsymbol{w}$ is the vector of Dial weights, whose generic element is $w_{d,(i, j)} ; \boldsymbol{\tau}$ is the vector of intensity of the pheromone trail, whose generic element is $\tau_{l}$.

Finally, D'Acierno et al. [1] stated the convergence of the proposed algorithm by means of the extension of Blum's theorem (Blum [9]) provided by Cantarella [7].

\section{The proposed assignment algorithm}

The aim of this paper is to extend ACO algorithms for solving the traffic assignment problem in the case of mass-transit networks by adopting the extension of Dial's algorithm (Dial [10]) proposed by Nguyen et al. [12]. Indeed, as Nguyen's algorithm is a network loading algorithm, it is necessary to introduce congestion assumptions (i.e. cost functions depending on network flows) in order to develop an assignment equilibrium algorithm. In the case of mass-transit systems, it is possible to identify three kinds of congestion: congestion due to the presence of other vehicles (i.e. buses in the case of exclusive lanes or cars and buses in the case of shared lanes); congestion of alighting and boarding links (due to alighting and boarding users) with related extension of dwelling times at bus stops; and congestion at bus stops due to bus capacity constraints.

Since the first two congestion types are very similar, in terms of cost functions, to the approach used by D'Acierno et al. [1] in the case of road systems, we propose to examine the third type. In the literature, congestion at bus stops due to bus capacity constraints can be modelled by means of two approaches:

- the semi-congested approach, which considers only the increase in user waiting times (Wu et al. [13]);

- the fully-congested approach, which also includes the effects on the flow split (Bouzaiene-Ayari et al. [14]).

In order to verify the practicability of an ACO approach in the case of mass-transit networks, we adopted the semi-congested approach that is less accurate but easier to implement. In particular, user waiting times at bus stops were formulated as:

$$
T_{j}^{w}=\left\{\begin{array}{cl}
\frac{\eta}{\sum_{n \in I_{j}} \varphi_{n}} & \text { if } R C_{j}>0 \text { and } f_{j}^{b}<R C_{j} \\
\frac{f_{j}^{b}}{R C_{j}} \cdot \frac{\eta}{\sum_{n \in I_{j}} \varphi_{n}} & \text { if } R C_{j}>0 \text { and } f_{j}^{b} \geq R C_{j}, \\
+\infty & \text { if } R C_{j} \leq 0
\end{array}\right.
$$


with:

$$
R C_{j}=\sum_{n \in I_{j}}\left(\varphi_{n} \cdot C a p_{n}\right)-f_{j, n}^{v}+f_{j, n}^{a} .
$$

where: $T_{j}^{w}$ is the waiting time at bus stop $j ; \eta$ is the service regularity term (whose value is between 0.5 and 1.0 ); $\varphi_{n}$ is the service frequency of line $n ; R C_{j}$ is the residual capacity at bus stop $j ; f_{j}^{b}$ is the boarding flow at bus stop $j ; f_{j}^{a}$ is the alighting flows at bus stop $j ; f_{j}^{v}$ is the in-vehicle flow just before bus stop $j$; Cap $_{n}$ is the vehicle capacity of line $n$ buses.

The framework of the algorithm proposed for applying the ACO approach in the case of mass-transit networks can be derived from the structure of the algorithm proposed by D'Acierno et al. [1] in the case of road networks. In particular, it is necessary to replace path costs in eqns (22) and (24) with hyperpath costs calculated as suggested by Nguyen et al. [12].

Therefore, adopting the theoretical proofs proposed by D'Acierno et al. [1], it is possible to state the perfect equivalence in terms of hyper-path user behaviour between artificial ants (described by the algorithm proposed in this paper) and real users (simulated by traditional traffic assignment algorithms).

Moreover, it is worth noting that the waiting time function proposed is not continuous and hence the theoretical properties of existence and uniqueness of the equilibrium solution cannot be stated. However, at least the existence of an equilibrium solution can be stated by adopting a different formulation of eqn (29) based on a continuous function, that is:

$$
T_{j}^{w}=\left\{\begin{array}{cl}
\frac{\eta}{\sum_{n \in I_{j}} \varphi_{n}} & \text { if } R C_{j}>0 \text { and } f_{j}^{b}<R C_{j} \\
\frac{f_{j}^{b}}{R C_{j}} \cdot \frac{\eta}{\sum_{n \in I_{j}} \varphi_{n}} & \text { if } R C_{j}>\varepsilon \text { and } f_{j}^{b} \geq R C_{j} \\
\left(\frac{f_{j}^{b}}{\varepsilon}+\frac{\varepsilon-R C_{j}}{\left(R C_{j}\right)^{2}}\right) \cdot \frac{\eta}{\sum_{n \in I_{j}} \varphi_{n}} & \text { if } R C_{j} \leq \varepsilon
\end{array} .\right.
$$

\section{First results}

The proposed algorithm was applied on a trial network (shown in Figure 1) which consists of two origin-destination pairs (101-103 and 102-103), one bus line with two transit links (11-12 and 12-13), two pedestrian links (1-2 and 2-3) and two boarding links (1-11 and 2-12).

Network features are indicated in Table 1. In particular, line capacity is equal to $600 \mathrm{users} / \mathrm{h}$ while at the second bus stop the residual capacity is equal to 50 seats and is lower than the boarding flows (450 users/ $h$ ). 


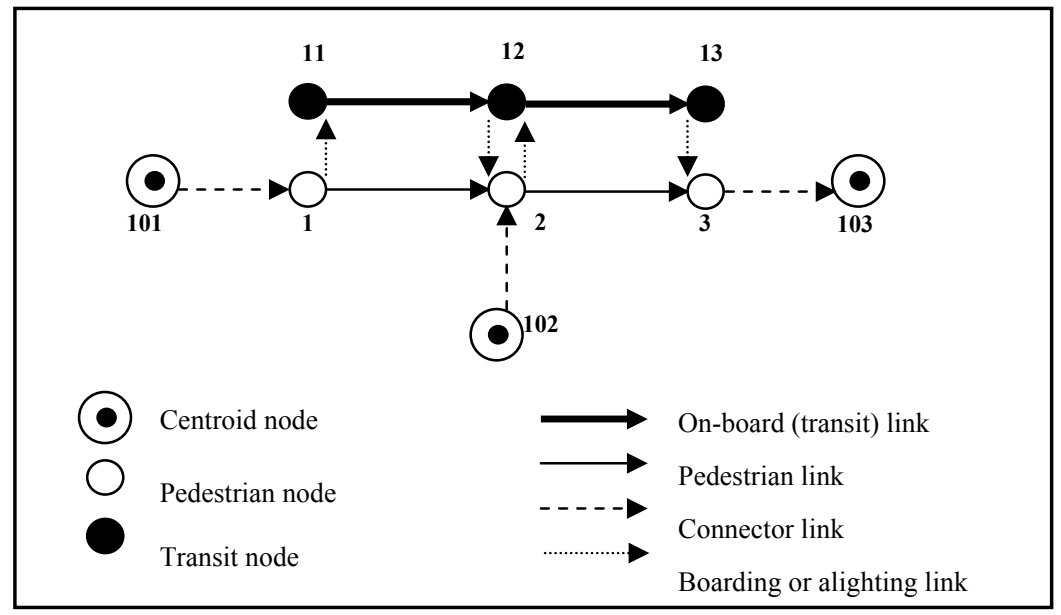

Figure 1: Trial network.

Table 1: $\quad$ Network features.

\begin{tabular}{cccccccc}
\hline \multicolumn{7}{c}{ Link performance } \\
\hline Link & $101-1$ & $102-2$ & $103-3$ & $1-2$ & $2-3$ & $11-12$ & $12-13$ \\
Travel time [min] & 10.0 & 10.0 & 10.0 & 20.0 & 20.0 & 8.0 & 8.0 \\
\hline Transit system & & & \multicolumn{5}{c}{ Travel demand } \\
\cline { 5 - 7 } Line & 1 & & Orig. & Destination & Value \\
Service frequency & 4 & & 101 & 103 & 550.0 \\
[buses./h] & & & 102 & 103 & 450.0 \\
& & & & &
\end{tabular}

Table 2: $\quad$ Algorithm performance.

\begin{tabular}{cccc}
\hline Algorithm name & Number of iterations & Convergence $(>90.0 \%)$ & Solution error \\
\hline$M S A-F A$ & 59 & 38 & Reference \\
$M S A-C A$ & 11 & 11 & $0.122 \%$ \\
$M S A-A C O$ & 7 & 7 & $0.169 \%$ \\
\hline
\end{tabular}

As indicated by Table 2 and Figure 2, the proposed algorithm (termed MSAACO) provides the same solution as the traditional algorithm. Indeed solution error is always lower than the stopping criterion set at $1.0 \%$.

Calculation times of the proposed algorithm, expressed by the number of iterations, are $88.14 \%$ lower than those of the MSA-FA (MSA-Flow Averaging) algorithm and $36.36 \%$ lower than that of the MSA-CA (MSA-Cost Averaging) algorithm.

If we assume that convergence is reached when more than $90 \%$ of links satisfy the stopping criterion (this assumption could be useful in the case of realscale networks), we obtain that the proposed algorithm requires calculation times $81.58 \%$ lower with respect to the MSA-FA algorithm and $36.36 \%$ lower than MSA-CA. 


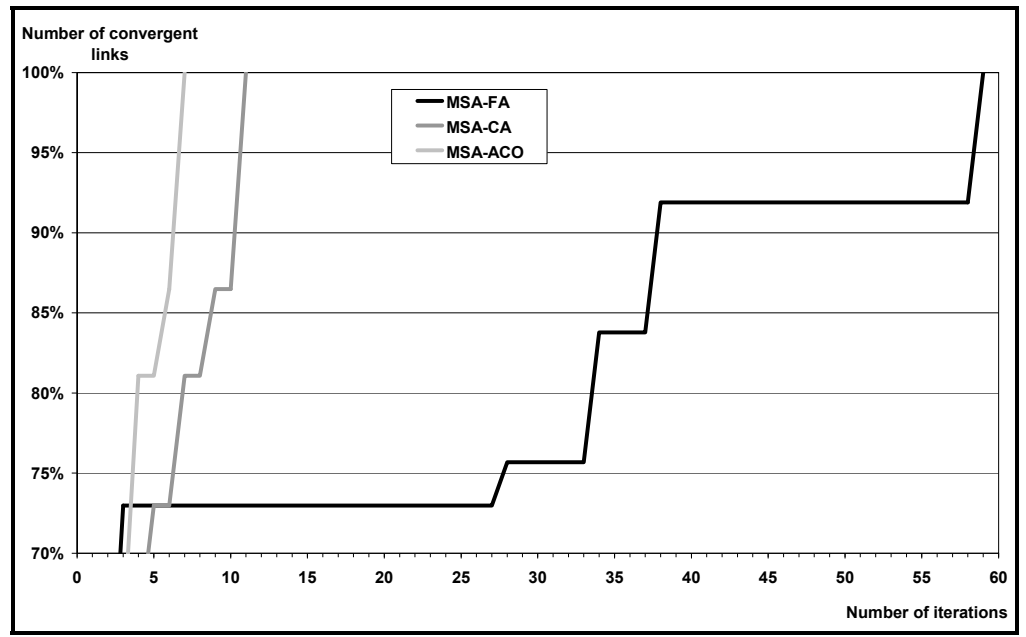

Figure 2: Algorithm performance.

\section{Conclusions and research prospects}

In this paper we proposed an ACO algorithm that can be utilised for solving the transit traffic assignment problem in the case of a line-based approach (i.e. a hyper-path approach). Moreover we showed its numerical efficiency with respect to traditional MSA algorithms.

In terms of future research, we suggest applying the proposed algorithm in the case of a real dimension network in order to verify whether its efficiency is retained. Moreover, we propose to extend the model in the case of a fullycongested approach and in the case of more complex hyper-path choice models (such as C-Logit and Probit).

Finally, the combination of the algorithm proposed in this paper (which allows mass-transit networks to be simulated) with that proposed by D'Acierno et al. [1] (which allows simulation of private car networks) could be used to develop multimodal assignment algorithms based on Ant Colony Optimisation, whose good performance could be exploited to speed up the search for solutions to complex problems such as the bus assignment problem in the urban context (D'Acierno et al. [15]), the urban freight distribution problem (D'Acierno et al. [16]), the multimodal design problem (Montella et al. [17]; D'Acierno et al. [18]), and the analysis of the AVL (Automatic Vehicle Location) data problem (D’Acierno et al. [19]).

\section{References}

[1] D’Acierno, L., Montella, B. \& De Lucia, F., A stochastic traffic assignment algorithm based on Ant Colony Optimisation. Lecture Notes in Computer Science 4150, pp. 25-36, 2006. 
[2] Dorigo, M. \& Stützle, T., Ant colony optimization. The MIT Press: Cambridge (MA), USA, 2004.

[3] Teodorovic, D., Swarm intelligence systems for transportation engineering: principles and applications. Transportation Research Part C, 16(6), pp. 651-667, 2008.

[4] Poorzahedy, H. \& Abulghasemi, F., Application of ant system to network design problem. Transportation, 32(3), 251-273, 2005.

[5] de Oliveira, D. \& Bazzan, A.L.C., Traffic lights control with adaptive group formation based on swarm intelligence. Lecture Notes in Computer Science 4150, pp. 520-521, 2006.

[6] Mussone, L. \& Matteucci, M., An application of Ant Colony Systems for DUE and SUE assignment in congested transportation networks. Proc. of the $11^{\text {th }}$ World Conference on Transport Research, Berkeley (CA), USA, 2007.

[7] Cantarella, G.E., A general fixed-point approach to multimodal multi-user equilibrium assignment with elastic demand. Transportation Science, 31(2), 107-128, 1997.

[8] Cascetta, E., Transportation systems engineering: theory and methods. Kluwer Academic Publisher: Dordrecht, The Netherlands, 2001.

[9] Blum, J.R., Multidimensional stochastic approximation methods. The Annals of Mathematical Statistics, 25(4), pp. 737-744, 1954.

[10] Dial, R.B., A probabilistic multipath traffic assignment model which obviates path enumeration. Transportation Research, 5(1), 83-111, 1971.

[11] Li, Y. \& Gong, S., Dynamic ant colony optimisation for TSP. The International Journal of Advanced Manufacturing Technology, 22(7-8), 528-533, 2003.

[12] Nguyen, S., Pallottino, S. \& Gendreau, M., Implicit enumeration of hyperpaths in a logit model for transit network. Transportation Science, 32(1), 54-64, 1998.

[13] Wu, J., Florian, M. \& Marcotte, P., Transit equilibrium assignment: a model and solution algorithms. Transportation Science, 28(3), 193-203, 1994.

[14] Bouzaiene-Ayari, B., Gendreau, M. \& Nguyen, S., Modeling bus stops in transit networks: a survey and new formulations. Transportation Science, 35(3), pp. 304-321, 2001.

[15] D’Acierno, L., Montella, B. \& Gallo, M., Multimodal assignment to congested networks: fixed-point models and algorithms. Proc. of the European Transport Conference 2002, Cambridge, United Kingdom, 2002.

[16] D’Acierno, L., Gallo, M. \& Montella, B., A fixed-point model and algorithms for simulating urban freight distribution in a multimodal context with crossed congestion. WIT Transactions on the Built Environment, 101, 589-598, 2008.

[17] Montella, B., Gallo, M. \& D’Acierno, L., Multimodal network design problems. Urban Transport $V$ - Urban Transport and the Environment of the $21^{\text {st }}$ Century, ed. L.J. Sucharov, WIT Press: Southampton, United Kingdom, 405-414, 2000. 
[18] D’Acierno, L., Gallo, M. \& Montella, B., Optimisation models for the urban parking pricing problem. Transport Policy, 13(1), 34-48, 2006.

[19] D'Acierno, L., Cartenì, A. \& Montella, B., Estimation of urban traffic conditions using an Automatic Vehicle Location (AVL) System. European Journal of Operational Research, 196(2), 719-736, 2009. 